Published in final edited form as:

Ann Biomed Eng. 2012 February ; 40(2): 367-377. doi:10.1007/s10439-011-0469-0.

\title{
Laser Speckle Contrast Imaging of Cerebral Blood Flow
}

\author{
Andrew K. Dunn \\ Biomedical Engineering Department University of Texas at Austin, Austin TX 78712
}

Andrew K. Dunn: adunn@mail.utexas.edu

\begin{abstract}
Laser speckle contrast imaging (LSCI) has emerged over the past decade as a powerful, yet simple, method for imaging of blood flow dynamics in real time. The rapid adoption of LSCI for physiological studies is due to the relative ease and low cost of building an instrument as well as the ability to quantify blood flow changes with excellent spatial and temporal resolution. Although measurements are limited to superficial tissues with no depth resolution, LSCI has been instrumental in pre-clinical studies of neurological disorders as well as clinical applications including dermatological, neurosurgical and endoscopic studies. Recently a number of technical advances have been developed to improve the quantitative accuracy and temporal resolution of speckle imaging. This article reviews some of these recent advances and describes several applications of speckle imaging.
\end{abstract}

\section{Introduction}

Methods for dynamic in vivo monitoring of blood flow are of great interest for a wide range of applications and diseases in both clinical and research settings. MRI, CT and ultrasound are the most common clinical methods. However, in research settings, optical techniques based on dynamic light scattering are the most common methods for blood flow monitoring such as laser Doppler, speckle contrast imaging and photon correlation spectroscopy [1]. Although each of these techniques differs in their measurement geometry and analysis, each is based on dynamic light scattering. Laser Doppler flowmetry is a well-established technique for measuring blood flow, although it is usually limited to measurements at single spatial locations. More recently, laser speckle contrast imaging (LSCI) has become widely used to image blood flow in a variety of tissues. Since LSCI enables full field imaging of surface blood flow using simple instrumentation, it has distinct advantages over techniques such as laser Doppler flowmetry. Although the instrumentation for LSCI is simple, obtaining quantitative measures of blood flow is very challenging due to the complex physics that relate the measured quantities to the underlying blood flow. This article reviews the physics of LSCI and illustrates some in vivo applications with particular emphasis on imaging blood flow in the brain. In addition, a new extension to traditional LSCI is described which is based on a multiple exposure technique, which improves the quantitative accuracy of speckle imaging of blood flow. Other recent review articles have covered different aspects and applications of speckle imaging and the reader is referred to those reviews as well [1], [2].

\section{Laser Speckle Imaging Physics}

When an object is illuminated with coherent laser light, a speckle pattern, or random interference pattern, is produced at the camera due to the fact that the laser light reaching each pixel has traveled slightly different pathlengths and adds coherently, both constructively and destructively. In many imaging systems speckle is a significant source of noise, and considerable effort has been spent in eliminating speckle. However, the dynamics 
of the speckle pattern contains information about the motion of the scattering particles in the sample. When some of the scattering particles are in motion (i. e. blood cells) the speckle pattern fluctuates in time. When the exposure time of the camera is longer than the time scale of the speckle intensity fluctuations (typically less than $1 \mathrm{~ms}$ for biological tissues), the camera integrates the intensity variations resulting in blurring of the speckle pattern. In areas of increased motion there is more blurring of the speckles during the camera exposure resulting in a lower spatial contrast of the speckles in these areas.

Full field imaging of blood flow based on laser speckle was first demonstrated by Fercher and Briers in 1981 [3], and this method was given the name LASCA (laser speckle contrast analysis). LASCA produced full field images of motion by quantifying the spatial contrast of a time-integrated speckle pattern. A significant limitation of early LASCA instruments however, was the need to record images on film and then process the images later, which resulted in low temporal resolution and large uncertainties in the speckle contrast values. With the advent of CCD cameras and modern computers in the 1990s, acquisition and processing of speckle images improved drastically and imaging of blood flow in tissues such as the skin and retina became possible.

Techniques based on the concept of time-integrated speckle rely on the fact that the motion of the scattering particles (i. e. blood flow) is encoded in the dynamics of the speckle pattern, and blood flow can be measured by quantifying the spatial blurring of the speckle pattern over the exposure time of the camera. The spatial blurring is measured by calculating the speckle contrast, $K$, defined as the ratio of the standard deviation, $\sigma_{\mathrm{s}}$, to the mean intensity of pixel values, $\langle I\rangle$, in a small region of the image [4],

$$
K(T)=\frac{\sigma_{s}(T)}{\langle I\rangle}
$$

where $T$ is the exposure time of the camera. The speckle contrast is therefore a measure of the local spatial contrast in the speckle pattern. A spatially resolved map of local speckle contrast can be calculated from a raw speckle image by computing this ratio at each point in the image from the pixels in a surrounding $N \mathrm{x} N$ region, (typically $N=7$ ). Theoretically the speckle contrast has values between 0 and 1 when the speckle pattern is sampled properly [5]. A speckle contrast of 1 indicates that there is no blurring of the speckle pattern and therefore, no motion, while a speckle contrast of 0 means that the scatterers are moving fast enough to average out all of the speckles.

One of the reasons that LSCI has become a widely adopted method for imaging blood flow is the relative simplicity of the instrumentation, as illustrated in Fig 1a. The basic configuration consists of a laser whose beam is expanded and adjusted to illuminate the area of interest, which can vary from a few millimeters to several centimeters. The angle of the incident light ranges from near normal incidence to as much as $45^{\circ}$, and the wavelength of the laser is generally in the red to near infrared region. The second main component is the camera. Light scattered from the sample is imaged onto the camera to enable recording of the speckle pattern. The specifications of the cameras used for LSCI vary widely, but inexpensive cameras have been demonstrated to provide excellent images of blood flow and enable detailed physiological studies to be performed [2], [6], [7]. In general, high dynamic range cooled cameras are not required for LSCI since the light levels reaching the camera are usually high enough that the majority of noise arises from shot noise. A wide range of wavelengths have also been used for LSCI, primarily throughout the red and near infrared regions. Since LSCI is based purely on scattering, the wavelength is significantly less important than imaging methods that rely on absorption contrast. Absorption is relatively 
low at wavelengths above $600 \mathrm{~nm}$ and diode lasers are widely available in this range. The different wavelengths will lead to slightly different sampling depths, but these differences are not large.

One significant limitation to LSCI is the shallow penetration depth of the images. Because of the illumination and detection geometry, detected photons sample only the superficial few hundred microns of tissue. As a result, most applications require removal of overlying tissues such as the skull in brain applications. Although monitoring of blood flow in the skin does not require any surgical access [8], LSCI is most commonly used in surgical settings [2], [6], [9].

A typical example of a raw speckle image of the rat cortex, taken through a thinned skull, and the computed speckle contrast are shown in Fig $1 \mathrm{~b}$ and $1 \mathrm{c}$ under normal conditions. The raw speckle image illustrates the grainy appearance of the speckle pattern. The speckle contrast image, computed directly from the raw speckle image using the Eq 1, represents a two-dimensional map of the magnitude of motion in the tissue, which is due primarily to blood flow. Areas of higher baseline flow, such as large vessels, have lower speckle contrast values and appear darker in the speckle contrast images. Fig 2 also illustrates the fact that LSCI is sensitive to blood flow in visible surface vasculature as well as areas of tissue that do not contain visible vasculature. In this example, the speckle contrast image in Fig 2a is taken from a mouse cortex through a cranial window. The same mouse was then imaged with multiphoton fluorescence microscopy after intravenous administration of a fluorophore to label the microvasculature. A maximum intensity projection of the multiphoton fluorescence image stack throughout the first $450 \mu \mathrm{m}$ of a portion of the LSCI field of view is shown in Fig $2 b$. These two images illustrate the fact that speckle contrast values in areas containing no obvious surface vasculature contain information about blood flow in the underlying microvasculature, even though the microvasculature is not spatially resolved. The speckle contrast values in areas lacking obvious surface vasculature are higher than areas containing vessels since overall blood flow is lower. However, changes in blood flow can still be detected in these areas [10], [11] and the speckle contrast increases drastically when flow is interrupted [12]. This effect is also evident in Fig 6, described in more detail below.

Although speckle contrast values are indicative of the level of motion in the sample, they are not directly proportional to speed or flow. In fact the exact quantitative relationship between speckle contrast and the underlying blood flow or speed is a complex function that is not completely understood for complex biological tissues [13]. In the early work of Briers, a simplified model was proposed that related the speckle contrast values to the speckle correlation time, $\tau_{\mathrm{c}}$ and speed of the scattering particles [3]. The intent of this model was not to establish a quantitative relationship between speckle contrast and speed, but to illustrate the relationship between time-integrated speckle and traditional techniques based on dynamic light scattering such as laser Doppler [1]. This simplified model has been demonstrated to be fairly accurate in its ability to predict relative changes in blood flow and has been used in a wide range of applications, particularly over the past several years.

Obtaining quantitative blood flow measurements from speckle contrast values involves two steps. The first is to accurately relate the speckle contrast values, which are obtained from a time integrated measure of the speckle intensity fluctuations, to a quantity commonly used in dynamic light scattering such as the speckle correlation time or a measure of the width of the Doppler power spectrum. The second step is to relate this quantity to the underlying flow or speed. The first step is unique to time integrated speckle measurements and has been the subject of several recent studies [13-15], while the second step is common to all dynamic 
light scattering techniques including laser Doppler, photon correlation spectroscopy and speckle techniques.

The relationship between the speckle contrast values, $K(T)$, and the speckle correlation decay time, $\tau_{\mathrm{c}}$, is rooted in the field of dynamic light scattering. The temporal fluctuations of the speckles can be quantified through the electric field autocorrelation function $g_{1}(\tau)$. Typical dynamic light scattering measurements involve measurement of the intensity autocorrelation function, $g_{2}(\tau)$. Using the Siegert relation, the field autocorrelation can be determined from the intensity autocorrelation,

$$
g_{2}(\tau)=1+\beta\left|g_{1}(\tau)\right|^{2}
$$

where $\beta$ is a normalization factor that accounts for speckle averaging due to the mismatch between speckle size and pixel size, as well as polarization effects and the finite coherence of the light source. The speckle correlation decay time, $\tau_{\mathrm{c}}$, is a measure of the decay time of the field autocorrelation function. The original relationship between $K(T)$ and $\tau \mathrm{c}$ formulated by Briers was

$$
K\left(T, \tau_{c}\right)=\left(\frac{1-e^{-2 x}}{2 x}\right)^{1 / 2}
$$

where $x=T / \tau_{\mathrm{c}}$. Although this relationship has been widely used in the literature, a more accurate expression that relates the measured speckle contrast to correlation decay time has been proposed [14],

$$
K\left(T, \tau_{c}\right)=\left(\beta \frac{e^{-2 x}-1+2 x}{2 x^{2}}\right)^{1 / 2}
$$

Generally LSCI measurements involve direct measurement of the speckle contrast at a single exposure time, and then conversion of the measured contrast to correlation decay time using either Eq 3 or 4 . Blood flow is then assumed to be inversely related to $\tau_{\mathrm{c}}$, and relative blood flow changes are determined by computing the changes in $\tau \mathrm{c}$ from some baseline state [16]. The assumption that blood flow is inversely proportional to $\tau c$ is based on a number of simplifying assumptions and is rooted in the laser Doppler literature [17]. Therefore, depending on the sample optical properties and flow conditions this assumption may become highly inaccurate. However, a number of reports have demonstrated a close agreement between speckle based measures of blood flow changes and laser Doppler flowmetry [12], [16]. Fig $2 \mathrm{c}$ illustrates a map of $\tau_{\mathfrak{c}}$ (colored regions) that was calculated from the speckle contrast image in Fig 2a using Eq 4.

Due to the large number of simplifying assumptions involved in Eqs 3 and 4, the quantitative accuracy of the measured blood flow changes may be low in some cases. Some groups have taken a different approach and do not attempt to relate the speckle contrast to correlation decay time. Instead, a calibration process is performed directly on the measured speckle contrast values [9], [18], and a quantity such as a perfusion index is reported. This procedure has the advantage that it does not rely on the assumptions that are built into Eq 3 and 4, but has the disadvantage that the values that are reported are not quantitative. 


\section{Multi-Exposure Laser Speckle Contrast Imaging (MESI)}

Despite the tremendous utility of LSCI for imaging blood flow in vivo, the method has some significant limitations. As described above single exposure LSCI is not able to quantify baseline, or absolute blood flow, and although LSCI is widely used to measure blood flow changes, the quantitative accuracy of these changes may be limited by the assumptions made in converting the measured speckle contrast values to speckle correlation times, $\tau_{\mathrm{c}}$. One of the factors that limits the accuracy of the blood flow changes measured with LSCI is the presence of light scattered from static tissue elements. Traditional models that relate speckle contrast to $\tau_{\mathrm{c}}$ do not take into account the presence of a static scattering layer such as the thinned or intact skull. Recently we have taken advantage of the dependence of the speckle contrast on camera exposure time to improve the accuracy of the $\tau_{\mathrm{c}}$ values [15]. This approach is called multi-exposure speckle contrast imaging (MESI) and it enables accurate determination of flow changes even in the presence of a static scattering layer. The MESI approach involves both a modified model and a modification to the experimental setup for single exposure LSCI.

Speckle contrast values are a strong function of the camera exposure time, $T$, as indicated in Eq 1. For very long exposure times, the speckle intensity fluctuations will have sufficient time to blur completely and therefore, speckle contrast values will approach 0 , while at very short exposure times the speckles will be frozen and contrast values will be high (in theory close to 1). Areas with lower flow result in speckle fluctuations that are slow compared to the exposure times and therefore, no appreciable blurring takes place. As the exposure time is increased, areas with lower flow, such as small vessels, appear in the speckle contrast image. Therefore, there is a close relationship between the exposure time and flow (or speed), and optimal sensitivity to different flow rates can be achieved by proper selection of exposure time [19]. MESI imaging capitalizes on the exposure time dependence of the speckle contrast to extract more accurate values of the speckle correlation times.

The MESI approach is a straightforward extension to the traditional theory relating $\tau_{\mathrm{c}}$ to $K(T)$, that accounts for the presence of static scattering. As discussed above, the field and intensity autocorrelation functions are related through the Siegert relation (Eq 2). However, in a sample with significant static scattering, the fluctuations in the scattered field acquire an extra static contribution that causes the recorded intensity to deviate from Gaussian statistics such that the Siegert relation cannot be applied [20], [21]. In this case a modified Siegert relation can be used of the form

$$
g_{2}^{h}(\tau)=1+A \beta\left|g_{1}(\tau)\right|^{2}+B \beta\left|g_{1}(\tau)\right|
$$

where $A=I_{f}^{2} /\left(I_{f}+I_{s}\right)^{2}$ and $B=2 I_{f} I_{s} /\left(I_{f}+I_{s}\right)^{2}$, and $I_{s}$ represents the intensity of the static scattered light and $I_{f}$ represents the intensity of dynamically scattered light.

This updated Siegert relation can be used to derive the relation between speckle contrast and speckle correlation time, $\tau_{\mathrm{c}}$, using the same approaches that have been used previously [14]. In addition, noise can be a significant contributor to the measured speckle contrast. Sources of noise include shot noise, statistical sampling noise, and camera noise, and each of these sources can vary with exposure time. Therefore the spatial variance of any given speckle image will contain contributions from dynamically scattered light, which is what we would like to quantify, as well as variance that arises from statically scattered light and noise sources. Recently Parthasarathy et al have derived a model that accounts for all of these components. Derivation of this model can be found in [22], and the result is a robust speckle contrast model that relates the measured speckle contrast to the speckle correlation time, 


$$
K\left(T, \tau_{c}\right)=\left(\beta \rho^{2} \frac{e^{-2 x}-1+2 x}{2 x^{2}}+4 \beta \rho(1-\rho) \frac{e^{-x}-1+x}{x^{2}}+v_{\text {noise }}\right)^{1 / 2}
$$

where $x=T / \tau_{c}, \rho=I_{f}\left(I_{f}+I_{S}\right)$ is the fraction of light that is dynamically scattered and $v_{\text {noise }}$ is the spatial variance that arises from all of the noise sources in the image such as camera noise and shot noise. Eq 6 therefore, represents an extension to the model of Eq 4 that accounts for both dynamic and static light scattering. In general, the parameters $\rho$ and $v_{\text {noise }}$ will be unknown for a given sample. Therefore, in order to use Eq 6, speckle contrast measurements must be acquired at multiple exposure times.

The instrumentation for MESI image acquisition requires control over both the camera exposure time and the laser intensity. To obtain speckle images at multiple exposure durations the actual camera exposure duration can be fixed while the laser diode is gated during each exposure to effectively vary the speckle exposure duration as in Yuan et. al. [19]. This approach ensures that the camera noise variance and the average image intensity are constant. Directly pulsing the laser limited the range of exposure durations that can be achieved. The lasing threshold of the laser diode dictated the minimum intensity and hence the maximum exposure duration that can be recorded. Consequently, the minimum exposure duration would be limited by the dynamic range of the instruments. To overcome this limitation the laser can begated by an acousto optic modulator (AOM). By modulating the amplitude of the RF wave fed to the AOM, the intensity of the first diffraction order can be varied, enabling control over both the integrated intensity and the effective exposure duration.

\section{Applications to Stroke}

LSCI has been used extensively in animal models of stroke because of its ability to provide real time images of the spatial and temporal blood flow dynamics. In focal ischemia blood flow is reduced in localized region of the brain leading to a cascade of cellular and molecular events that ultimately results in tissue damage. Blood flow in the area closest to the affected region (ischemic core) is typically reduced to less than $20 \%$ of its baseline value, and as a result neurons depolarize and die rapidly. In the region between the ischemic core and the normal tissue (penumbra), neurons preserve the ability to maintain ion homeostasis but are considered to be electrically silent since evoked potentials and spontaneous electrical activity cease [23]. The reduction in blood flow in the penumbra is not as severe as in the core since collateral blood supply to the area is maintained. This reduction in flow in the penumbra can lead to secondary effects such as peri-infarct spreading depolarizations, inflammation, and ultimately apoptosis [24]. Therefore, the ability to dynamically image the ischemic penumbra with good spatial resolution using simple instrumentation has been a major factor in the widespread use of LSCI in acute stroke studies.

The penumbra has been a primary target of treatment strategies since membrane function is preserved in cells in the penumbra. Restoration of blood flow to the penumbra therefore, may provide a means of salvaging tissue without loss of function. One of the mechanisms that may lead to cell death in the penumbra is the presence of peri-infarct depolarizations (PID) which resemble spreading depressions in the normal brain [24], [25]. Due to increased extracellular levels of $\mathrm{K}+$ and glutamate in the penumbra, cells depolarize [26]. These cells are able to repolarize, but at the expense of ATP. Gradually the cells in the penumbra lose the ability to repolarize due to the depletion of energy, leading to cell death. As the frequency of PID's increases, the extent of the ischemic injury has been found to increase as well [24], [27], [28]. Therefore, survival of the penumbra depends on the balance between 
CBF and metabolic demand. Although the metabolic and electrophysiologic consequences of these events during stroke have been extensively investigated, their impact on cerebral vasculature and CBF are less known. Recently several studies have investigated the role of spreading depolarizations in the expansion of the ischemic infarct using laser speckle contrast imaging in both rodent and cat models [27], [29], [30]. These studies indicated that the hemodynamic and metabolic response to PID's in the ischemic brain varies across the ischemic territory and each event leads to a stepwise expansion of the size of the ischemic territory.

An important consideration in speckle imaging during stroke is the quantitative accuracy of the blood flow changes. Since the size of the ischemic infarct is sometimes defined in terms of the area of tissue whose blood flow deficit is below a certain threshold [29], the blood flow changes must be accurate. Recently, a comparison was performed between traditional single exposure LSCI and MESI in a stroke model [31], and the results demonstrated that MESI is considerably more accurate in quantifying blood flow changes. To perform these studies a variable craniotomy model was developed in which certain parts of the field of view contained a thinned skull while other areas consisted of a full removal of the skull (Fig 3). MESI measurements of speckle contrast as a function of exposure time on either side of the variable craniotomy reveal considerable differences in the speckle contrast values at most exposure times (Fig 3). Since actual blood flow is expected to be comparable at each pair of locations, single exposure LSCI would likely result in inaccurate blood flow estimates. Furthermore, the shape of the speckle contrast curve with exposure time differs between regions, illustrating the importance of the MESI measurements combined with the MESI model.

The variable craniotomy model was combined with a photothrombotic stroke model which allowed a single vessel that spanned both areas of the craniotomy to be occluded. Therefore, the resulting blood flow deficit is expected to be similar on both sides of the craniotomy. MESI measurements were acquired during vessel occlusion and the speckle correlation times were determined by fitting the MESI data to Eq 6, and by using a single exposure measurement and converting to $\tau_{\mathrm{c}}$ using Eq 4 . The comparison of MESI and single exposure LSCI (Fig 3) reveals that the single exposure estimates of the flow changes following vessel occlusion are inconsistent across adjacent areas of the craniotomy. In some cases the flow values in adjacent pairs vary by more than $50 \%$. The MESI measurements on the other hand reveal consistent flow values for each pair of locations. The regions located directly over a larger blood vessel reveal the most prominent differences between MESI and single exposure LSCI. These results are particularly important since they demonstrate that MESI should lead to more consistent estimates of infarct sizes when measured by area of tissue below a particular blood flow threshold.

\section{Real-Time Intraoperative Imaging of Blood Flow}

Although the majority of speckle imaging applications in the brain have been in animal models, a few recent studies have investigated the use of speckle imaging in humans during neurosurgery [32], [33]. Monitoring of blood flow is important in most types of neurosurgical procedures including tumor resection, aneurysm repair and epilepsy surgery. However, surgeons rarely monitor perfusion continuously due to a lack of suitable methods for real-time assessment of blood flow. Although methods based on MRI and CT can be used, they disrupt the surgical procedure and require significant additions of time to the procedure. Over the past several years indocyanine green (ICG) angiography has been developed as an effective method for determining vessel perfusion intraoperatively [34]. ICG angiography entails intravenous administration of ICG, which is a near infrared fluorophore, and illumination of the surgical field with near infrared excitation light. The 
resulting fluorescence is imaged onto a camera that is built into the surgical operating microscope. Perfused vessels become fluorescent while blocked vessels remain dark. Therefore, ICG angiography is effective at identifying blocked vessels and does not require disruption to the surgical procedure since all imaging is performed with the operating microscope. However, the need to administer a contrast agent limits the use of ICG angiography to only a few measurements per surgery. In addition, it is very difficult to obtain quantitative flow values from ICG angiography measurements so surgeons most often use ICG angiography as a binary flow/no flow assessment.

More recently, LSCI has been used to image blood flow during neurosurgery [32], [33]. Unlike ICG angiography, LSCI does not require an exogenous contrast agent and as a result, the number of measurements is unlimited. When the LSCI instrumentation is built into the surgical operating microscope (Fig 4), neurosurgeons can easily visualize blood flow simply by turning the laser source on and off [33]. An important consideration for clinical use of LSCI is real-time display of blood flow images. Several groups have investigated the use of graphical processing units (GPU) to accelerate the calculation of speckle contrast in order to achieve near real-time imaging rates [35]. However, highly optimized implementation of the speckle contrast calculation can enable processing rates that exceed image acquisition rates using standard computer hardware. In fact, using the calculation approach developed by Tom et al [36], processing rates of more than 600 frames/sec for full field images (656x491 pixels) can be achieved with an inexpensive laptop computer. Therefore, the processing times are no longer the limiting factor in imaging speeds for LSCI. Instead imaging speed is limited by the acquisition rate of the camera.

LSCI has been demonstrated to be sensitive to blood flow changes in the human brain [33]. In addition, LSCI is very sensitive to the pulsatile motion of the brain during neurosurgery. Since the speckle contrast is inherently sensitive to motion, the pulsatile motion of the exposed cortex induces temporal variations in the speckle contrast that add to the blood flow induced speckle contrast variations. Because the pulsatile motion is fairly regular with the cardiac cycle, ad hoc filters can be created by examining the speckle contrast variations within a normalized cardiac cycle [33]. This filter can be applied to the speckle contrast timecourse to significantly reduce noise in the speckle contrast due to pulsatile motion (Fig $5)$.

\section{Combined Imaging of Blood Flow and Oxygen Tension}

In addition to blood flow, other hemodynamic parameters such as hemoglobin oxygenation and volume are frequently of interest. Because LSCI and MESI measurements are relatively insensitive to laser wavelength, they can be combined with other optical techniques that utilize different wavelengths to achieve simultaneous imaging of blood flow and other hemodynamic parameters [37]. Combined imaging of blood flow and hemoglobin oxygenation has been used to investigate cerebral hemodynamics during stroke [29], [38], cortical spreading depression [37], and functional brain activation [7], [37].

More recently, LSCI has been combined with phosphorescence lifetime quenching methods to simultaneously image blood flow and oxygen tension $\left(\mathrm{pO}_{2}\right)$ [39], [40]. In vivo measurement of $\mathrm{pO}_{2}$ has been accomplished using several different methods including oxygen sensitive electrodes, oxygen dependent phosphorescence quenching and electron paramagnetic resonance [41]. Oxygen sensitive electrodes can provide $\mathrm{pO}_{2}$ measurements from small tissue volumes due to the small size of many electrodes, but suffer from the fact that they are single point measurements and they are invasive and can lead to tissue damage. Determination of $\mathrm{pO}_{2}$ using phosphorescence quenching has been shown to be highly effective for in vivo measurements due to recent advances in imaging technology and probe 
design [42-46]. This method is based on the fact that dissolved oxygen quenches the phosphorescence or fluorescence of certain compounds such as porphyrin dyes and ruthenium complexes, resulting in changes in excited state lifetimes. As a result, the $\mathrm{pO}_{2}$ can be quantified from the measured lifetime using the Stern-Volmer relationship

$$
\frac{\tau_{o}}{\tau}=1+k_{q} \tau_{o}\left[\mathrm{pO}_{2}\right]
$$

where $\tau$ is the measured lifetime, $\tau_{\mathrm{o}}$ is the unquenched lifetime and $k_{q}$ is a quenching constant that depends on the properties of the dye and the surrounding environment. Therefore absolute oxygenation can be quantified from the measured decay time provided that the quenching constant and unquenched lifetime are known. In addition, lifetime based measurements are not dependent on absolute intensities, which eliminates the need to correct for the presence of other tissue chromophores or tissue scattering.

Despite its widespread use, phosphorescence quenching methods are almost always limited to a single spatial location and only a few reports have demonstrated $\mathrm{pO}_{2}$ imaging or spatial mapping [39], [40], [47-49]. This limitation arises from the need to resolve the phosphorescence lifetime, which makes most cameras extremely difficult to use. Single point detectors such as avalanche photodiodes and photomultiplier tubes are significantly more sensitive and have sufficient temporal resolution to resolve the phosphorescence decay times.

In order to obtain spatially resolved $\mathrm{pO}_{2}$ measurements, we have utilized a digital micromirror device combined with a sensitive single element detector such as a photomultiplier or avalanche photodiode [40]. This approach retains the high sensitivity of single element detectors while still permitting spatially resolved phosphorescence decay measurements (Fig 6). A speckle contrast image is first obtained, and several regions of interest are selected from the image of the surface vasculature. Each region is transformed to the DMD coordinates and the appropriate mirrors of the DMD are turned on to restrict the pulsed phosphorescence excitation light to this region. The entire phosphorescence emission from that region is collected by the detector and the decay time is determined and converted to a $\mathrm{pO}_{2}$ value. The entire system is controlled by custom software and the DMD switches illumination regions at approximately 10 regions per second.

An example of the use of this system in a rodent stroke model is illustrated in Fig $6 \mathrm{~d}-\mathrm{f}$. In this example, 8 spatial regions were selected for $\mathrm{pO}_{2}$ measurement. The blood flow (Fig 6e) and $\mathrm{pO}_{2}$ (Fig 6f) timecourses over the 8 regions demonstrate that the changes in these two parameters during the course of ischemia differ, particularly in branches of the arteriole. In particular, the CBF drop is larger than the corresponding decrease in $\mathrm{pO}_{2}$. This example illustrates the great potential of combined optical imaging instruments for revealing the subtelties of hemodynamic changes in the cortex during cerebrovascular pathologies.

\section{Conclusions}

Laser speckle contrast imaging is a powerful method for real-time visualization of blood flow. Its simple instrumentation and low cost make LSCI readily accessible for many applications ranging from basic physiology to clinical settings. Traditionally LSCI has been limited to measurements of relative blood flow changes within a single animal. Recent advances such as multi-exposure speckle imaging however, should enable chronic imaging of blood flow as well as improve the quantitative accuracy of blood flow imaging. In addition, speckle imaging combined with other optical imaging modalities should enable simultaneous multi-parameter imaging of a range of physiological parameters. 


\section{Acknowledgments}

The author acknowledges Shams Kazmi, Adrien Ponticorvo, Lisa Richards, Erica Weber, Ashwin Parthasarathy, and Anthony Salvaggio for helpful discussions and assistance with the figures. This work was supported by the National Institutes of Health (R01EB011556), National Science Foundation (CBET/0737731), American Heart Association $(0735136 \mathrm{~N})$ and the Coulter Foundation.

\section{References}

1. Briers JD. Laser Doppler, speckle and related techniques for blood perfusion mapping and imaging. Physiol Meas. 2001; 22(4):R35-66. [PubMed: 11761081]

2. Boas DA, Dunn AK. Laser speckle contrast imaging in biomedical optics. Journal of biomedical optics. 2010; 15(1):011109. [PubMed: 20210435]

3. Fercher A, Briers J. Flow visualization by means of single-exposure speckle photography. Opt Commun. 1981; 37:326-329.

4. Briers JD, Webster S. Laser Speckle contrast analysis \{(LASCA): \} A nonscanning, full-field technique for monitoring capillary blood flow. Journal of Biomedical Optics. 1996; 1:174-179.

5. Kirkpatrick SJ, Duncan DD, Wells-Gray EM. Detrimental effects of speckle-pixel size matching in laser speckle contrast imaging. Optics Letters. Dec; 2008 33(24):2886-2888. [PubMed: 19079481]

6. Bolay H, Reuter U, Dunn AK, Huang Z, Boas DA, Moskowitz MA. Intrinsic brain activity triggers trigeminal meningeal afferents in a migraine model. Nature Medicine. 2002; 8(2):136-42.

7. Dunn AK, Devor A, Dale AM, Boas DA. Spatial extent of oxygen metabolism and hemodynamic changes during functional activation of the rat somatosensory cortex. Neuroimage. $2005 ; 27(2)$ : 279-290. [PubMed: 15925522]

8. Huang Y-C, Ringold TL, Nelson JS, Choi B. Noninvasive blood flow imaging for real-time feedback during laser therapy of port wine stain birthmarks. Lasers in Surgery and Medicine. Mar; 2008 40(3):167-173. [PubMed: 18366081]

9. Forrester KR, Tulip J, Leonard C, Stewart C, Bray RC. A laser speckle imaging technique for measuring tissue perfusion. \{IEEE\} Trans Biomed Eng. 2004; 51(11):2074-2084.

10. Durduran T, et al. Spatiotemporal quantification of cerebral blood flow during functional activation in rat somatosensory cortex using laser-speckle flowmetry. J Cereb Blood Flow Metab. 2004; 24(5):518-525. [PubMed: 15129183]

11. Dunn AK, Devor A, Dale AM, Boas DA. Spatial extent of oxygen metabolism and hemodynamic changes during functional activation of the rat somatosensory cortex. Neuroimage. $2005 ; 27(2)$ : 279-290. [PubMed: 15925522]

12. Ayata C, Dunn AK, Gursoy OzY, Huang Z, Boas DA, Moskowitz MA. Laser speckle flowmetry for the study of cerebrovascular physiology in normal and ischemic mouse cortex. J Cereb Blood Flow Metab. 2004; 24(7):744-755. [PubMed: 15241182]

13. Duncan DD, Kirkpatrick SJ. Can laser speckle flowmetry be made a quantitative tool? Journal of the Optical Society of America A, Optics, Image Science, and Vision. Aug; 2008 25(8):20882094.

14. Bandyopadhay R, Gittings AS, Suh SS, Dixon PK, Durian DJ. Speckle-visibility spectroscopy: A tool to study time-varying dynamics. Review of Scientific Instruments. 2005; 76(9):93110.

15. Parthasarathy AB, Tom WJ, Gopal A, Zhang X, Dunn AK. Robust flow measurement with multiexposure speckle imaging. Optics Express. Feb; 2008 16(3):1975-1989. [PubMed: 18542277]

16. Dunn AK, Bolay H, Moskowitz MA, Boas DA. Dynamic imaging of cerebral blood flow using laser speckle. Journal of Cerebral Blood Flow \& Metabolism. 2001; 21(3):195-201. [PubMed: 11295873]

17. Bonner R, Nossal R. Model for laser Doppler measurements of blood flow in tissue. Applied Optics. 1981; 20:2097-2107. [PubMed: 20332893]

18. Tamaki Y, Araie M, Kawamoto E, Eguchi S, Fujii H. Noncontact, two-dimensional measurement of retinal microcirculation using laser speckle phenomenon. Invest Ophthalmol Vis Sci. Oct; 1994 35(11):3825-3834. [PubMed: 7928179] 
19. Yuan S, Devor A, Boas DA, Dunn AK. Determination of optimal exposure time for imaging of blood flow changes with laser speckle contrast imaging. Appl Opt. 2005; 44(10):1823-1830. [PubMed: 15813518]

20. Lemieux PA, Durian DJ. Investigating \{non-Gaussian\} scattering processes by using $\mathrm{n}$ th-order intensity correlation functions. Journal of the Optical Society of America A. 1999; 16(7):16511664.

21. Boas DA, Yodh A. Spatially Varying Dynamical Properties of Turbid Media Probed with Diffusing Temporal Light Correlation. Journal of the Optical Society of America A. 1997; 14:192-215.

22. Parthasarathy AB, Tom WJ, Gopal A, Zhang X, Dunn AK. Robust flow measurement with multiexposure speckle imaging. Optics Express. Feb; 2008 16(3):1975-1989. [PubMed: 18542277]

23. Lo EH, Dalkara T, Moskowitz MA. Mechanisms, challenges and opportunities in stroke. Nature Reviews Neuroscience. May; 2003 4(5):399-415.

24. Mies G, Iijima T, Hossmann KA. Correlation between peri-infarct $\{\mathrm{DC}\}$ shifts and ischaemic neuronal damage in rat. Neuroreport. 1993; 4:709-711. [PubMed: 8347812]

25. Hossmann KA. Periinfarct depolarizations. Cerebrovasc Brain Metab Rev. 1996; 8:195-208. [PubMed: 8870974]

26. Dirnagl U, Iadecola C, Moskowitz MA. Pathobiology of ischaemic stroke: an integrated view. Trends in Neuroscience. 1999; 22:391-397.

27. Shin HK, Dunn AK, Jones PB, Boas DA, Moskowitz MA, Ayata C. Vasoconstrictive neurovascular coupling during focal ischemic depolarizations. J Cereb Blood Flow Metab. 2005

28. Strong AJ, Bezzina EL, Anderson PJ, Boutelle MG, Hopwood SE, Dunn AK. Evaluation of laser speckle flowmetry for imaging cortical perfusion in experimental stroke studies: quantitation of perfusion and detection of peri-infarct depolarisations. J Cereb Blood Flow Metab. 2006; 26(5): 645-653. [PubMed: 16251884]

29. Shin HK, et al. Normobaric hyperoxia improves cerebral blood flow and oxygenation, and inhibits peri-infarct depolarizations in experimental focal ischaemia. Brain. 2007; 130(6):1631. [PubMed: 17468117]

30. Nakamura H, et al. Spreading depolarizations cycle around and enlarge focal ischaemic brain lesions. Brain. Jul; 2010 133(7):1994-2006. [PubMed: 20504874]

31. Parthasarathy AB, Kazmi SMS, Dunn AK. Quantitative imaging of ischemic stroke through thinned skull in mice with Multi Exposure Speckle Imaging. Biomedical optics express. Jan; 2010 1(1):246-259. [PubMed: 21258462]

32. Hecht N, Woitzik J, Dreier JP, Vajkoczy P. Intraoperative monitoring of cerebral blood flow by laser speckle contrast analysis. Neurosurgical focus. Oct.2009 27(4):E11. [PubMed: 19795950]

33. Parthasarathy AB, Weber EL, Richards LM, Fox DJ, Dunn AK. Laser speckle contrast imaging of cerebral blood flow in humans during neurosurgery: a pilot clinical study. Journal of Biomedical Optics. Jan.2010 15(6):066030. [PubMed: 21198204]

34. Raabe A, Beck J, Gerlach R, Zimmermann M, Seifert V. Near-infrared indocyanine green video angiography: a new method for intraoperative assessment of vascular flow. Neurosurgery. 2003; 52(1):132-9. discussion 139. [PubMed: 12493110]

35. Liu S, Li P, Luo Q. Fast blood flow visualization of high-resolution laser speckle imaging data using graphics processing unit. Optics express. Sep; 2008 16(19):14321-9. [PubMed: 18794967]

36. Tom WJ, Ponticorvo A, Dunn AK. Efficient processing of laser speckle contrast images. \{IEEE\} Transactions on Medical Imaging. Dec; 2008 27(12):1728-1738.

37. Dunn AK, et al. Simultaneous imaging of total cerebral hemoglobin concentration, oxygenation, and blood flow during functional activation. Optics Letters. 2003; 28:28-30. [PubMed: 12656525]

38. Jones PB, et al. Simultaneous multispectral reflectance imaging and laser speckle flowmetry of cerebral blood flow and oxygen metabolism in focal cerebral ischemia. Journal of Biomedical Optics. Jul; 2008 13(4):44007-44011.

39. Sakadzić S, et al. Simultaneous imaging of cerebral partial pressure of oxygen and blood flow during functional activation and cortical spreading depression. Applied Optics. Apr; 2009 48(10):D169-177. [PubMed: 19340106] 
40. Ponticorvo A, Dunn AK. Simultaneous imaging of oxygen tension and blood flow in animals using a digital micromirror device. Optics express. Apr; 2010 18(8):8160-70. [PubMed: 20588661]

41. Tsai A, Johnson PC, Intaglietta M. Oxygen Gradients in the Microcirculation. Physiological Reviews. 2003; 83(3):933-963. [PubMed: 12843412]

42. Rumsey WL, Vanderkooi JM, Wilson DF. Imaging of phosphorescence: a novel method for measuring oxygen distribution in perfused tissue. Science. 1988; 241(4873):1649. [PubMed: 3420417]

43. Shonat RD, Wilson DF, Riva CE, Pawlowski M. Oxygen distribution in the retinal and choroidal vessels of the cat as measured by a new phosphorescence imaging method. Appl Opt. 1992; 31(19):3711-3718. [PubMed: 20725344]

44. Shonat RD, Richmond KN, Johnson PC. Phosphorescence quenching and the microcirculation: An automated, multipoint oxygen tension measuring instrument. Review of Scientific Instruments. 1995; 66:5075.

45. Vinogradov SF-SMA, Dugan BW, Wilson DF. Frequency domain instrument for measuring phosphorescence lifetime distributions in heterogeneous samples. Review of Scientific Instruments. 2001; 7(8):3396-3406.

46. Dunphy I, Vinogradov SA, Wilson DF. Oxyphor R2 and G2: phosphors for measuring oxygen by oxygen-dependent quenching of phosphorescence. Analytical Biochemistry. 2002; 310(2):191198. [PubMed: 12423638]

47. Shonat RD, Kight AC. Oxygen tension imaging in the mouse retina. Ann Biomed Eng. 2003; 31(9):1084-1096. [PubMed: 14582611]

48. Shonat RD, Wachman ES, Niu W, Koretsky AP, Farkas DL. Near-simultaneous hemoglobin saturation and oxygen tension maps in mouse brain using an $\{$ AOTF $\}$ microscope. Biophys $\mathrm{J}$. 1997; 73(3):1223-1231. [PubMed: 9284290]

49. Helmlinger G, Yuan F, Dellian M, Jain RK. Interstitial $\{\mathrm{pH}\}$ and $\{\mathrm{pO}\} 2$ gradients in solid tumors in vivo: High-resolution measurements reveal a lack of correlation. Nature Medicine. 1997; 3(2): 177-182. 

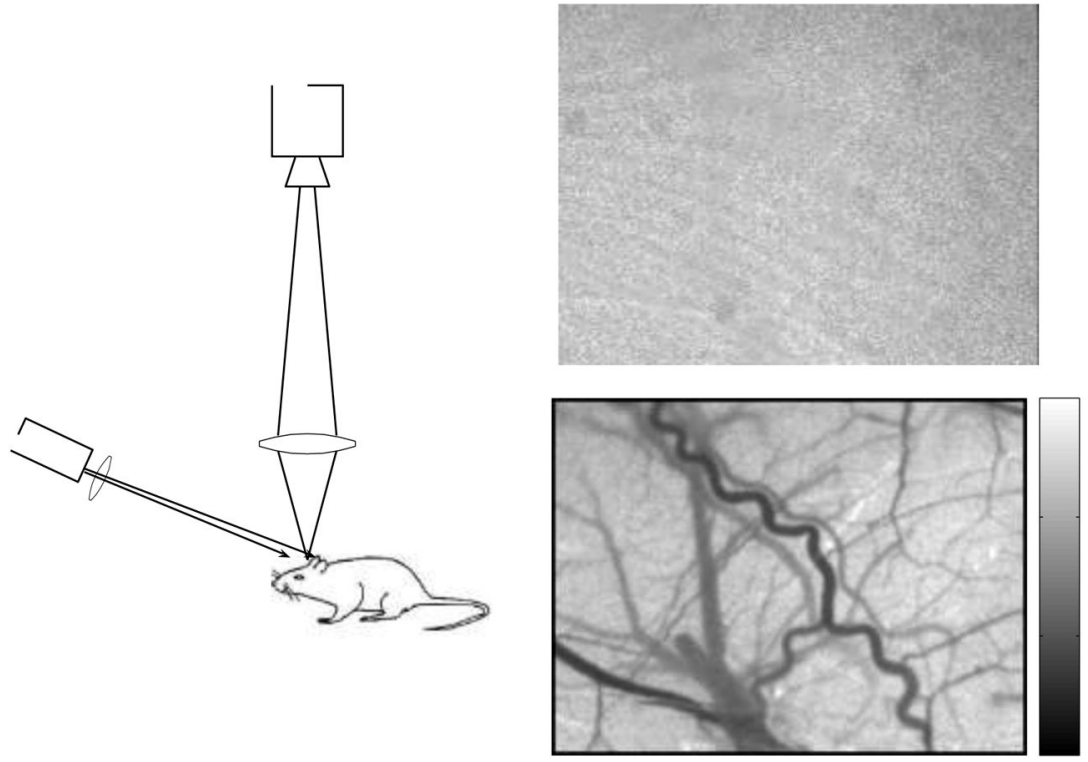

Figure 1.

(a) Basic laser speckle contrast imaging setup consisting of a laser diode and camera. A raw speckle image of the rat cortex, taken through a thinned skull, contains seemingly little information (b). However, when the speckle contrast is calculated using a sliding window (c), a tremendous amount of information is revealed about the motion of the scattering particles in the sample. 

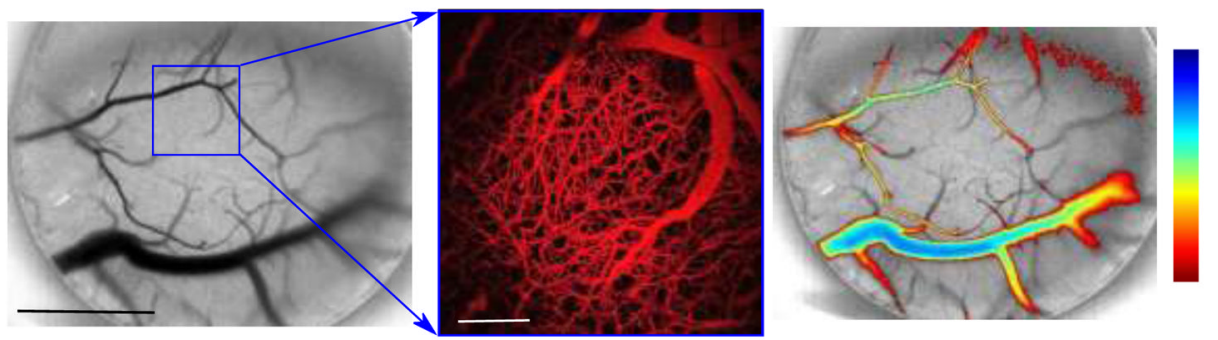

Figure 2.

(a) Speckle contrast image of mouse cortex taken though a cranial window. A maximum intensity projection of a multiphoton fluorescence image stack (b) of the area highlighted by the blue square in (a) indicates that even in areas of the speckle contrast images that contain no obvious surface vasculature, the speckle contrast values contain contributions from subsurface microvasculature. The relative amounts of this subsurface contribution will vary depending on tissue type and surface preparation. Note that only the right portion of the surface vessel that is visible in the speckle image (a) is observed in the multiphoton fluorescence image stack since the image stack began at a depth slightly below the left hand portion of the vessel. The image in (c) shows a color coded map of $\tau_{\mathrm{c}}$ values from a mouse cortex where a threshold has been applied to highlight flow within different vascular compartments. 

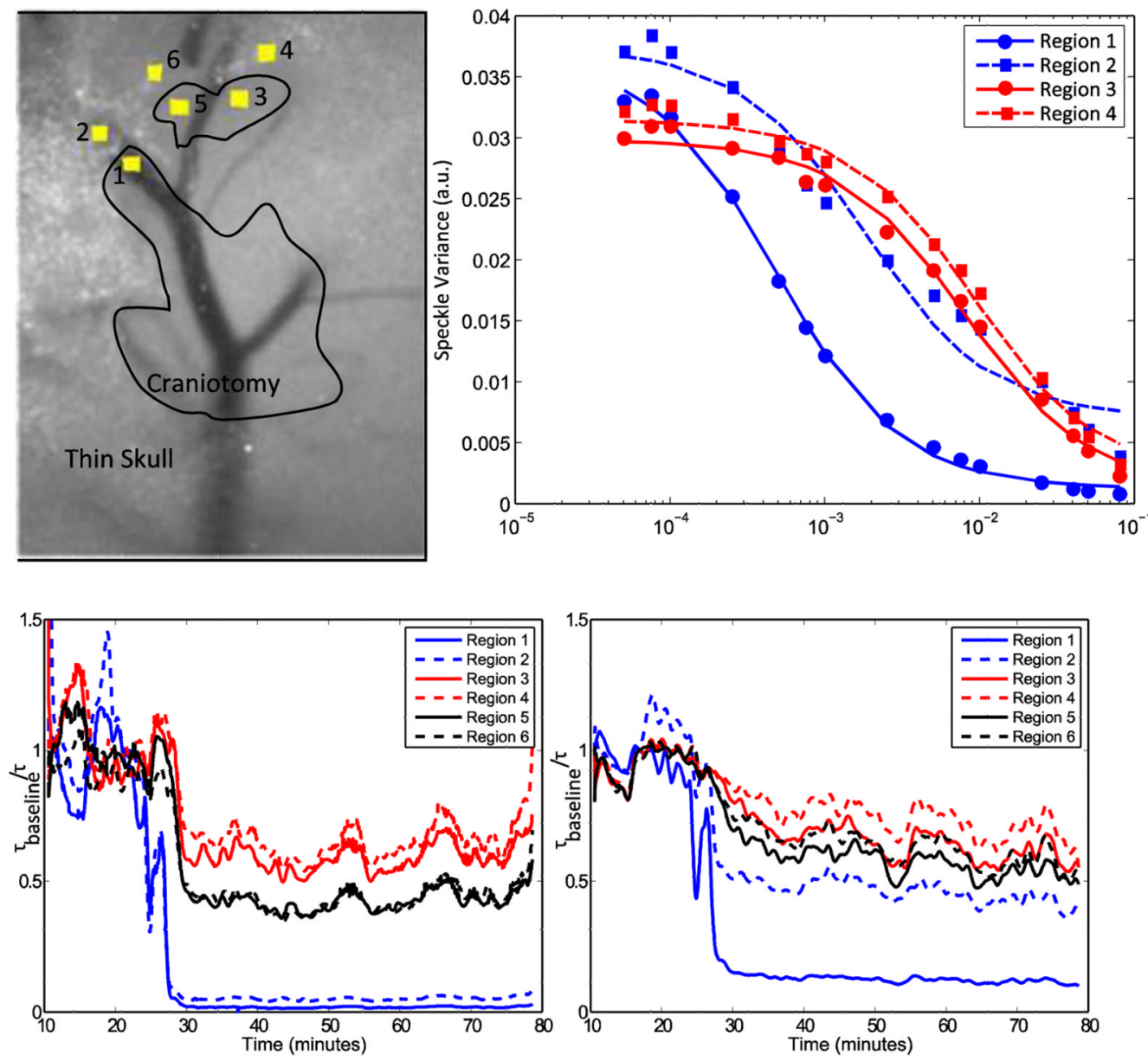

Figure 3.

Multi-exposure speckle imaging during acute stroke. A variable thickness craniotomy model (a) enabled in vivo evaluation of the effect of skull thickness on flow changes. A photothrombotic stroke model was used to occlude a single vessel and MESI and single exposure LSCI images were acquired throughout the vessel occlusion. The fits of the MESI data to Eq 6 reveal strong differences in the shape of $K(T)$ in different regions of the tissue (b). The flow changes on adjacent locations of the craniotomy borders during the first 80 minutes of stroke are very consistent for MESI measurements (c). However, single exposure LSCI analysis leads to significant differences in the predicted flow changes between adjacent areas (d). The values $\tau_{\text {baseline }}$ and $\tau$ represent $\tau_{\mathrm{c}}$ at different time points. 

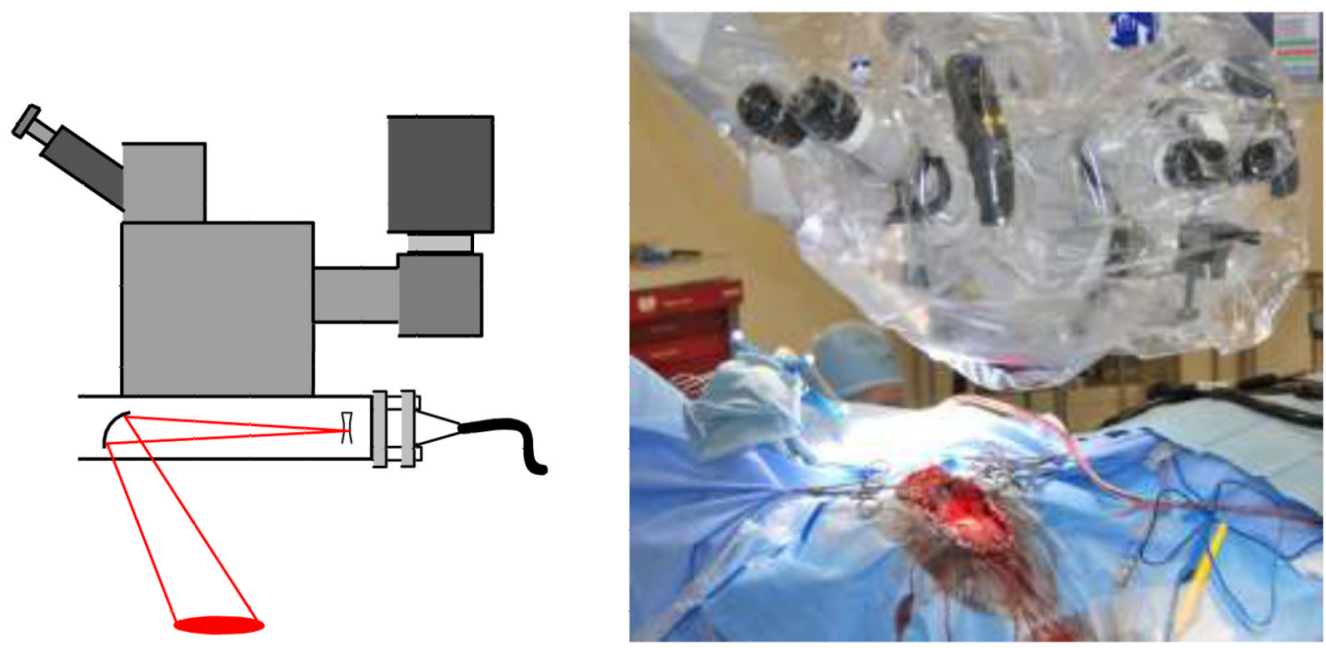

Figure 4.

Schematic and photograph of neurosurgical operating microscope adapted for real-time speckle imaging of blood flow during neurosurgery. A commercially available laser illuminator was fitted with a laser diode $(660 \mathrm{~nm})$ and a camera was attached to an existing camera port on the microscope, which enabled all of the instrumentation to be contained within the surgical drape. 


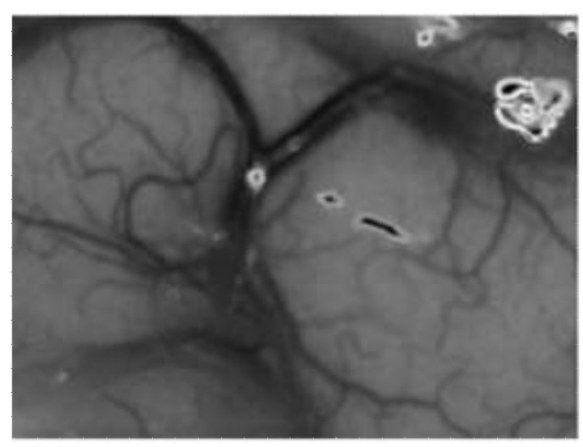

(a)

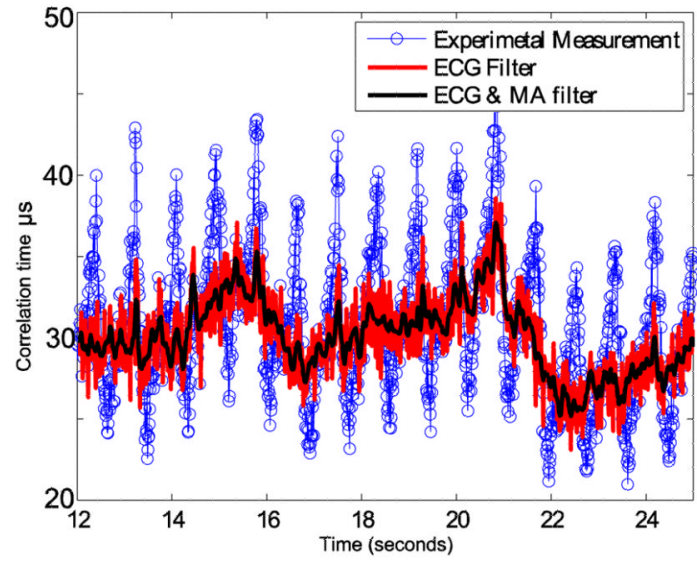

(b)

Figure 5.

(a) Speckle contrast image of human cortex averaged over 30 LSCI images. Cardiac and respiratory motion of the exposed cortex leads to temporal variations in speckle contrast values since LSCI is inherently sensitive to motion (b). A simple filter based on the camera acquisition times within the cardiac cycle is effective at removing noise in the speckle contrast signal, particularly when combined with a moving average (MA) filter. 

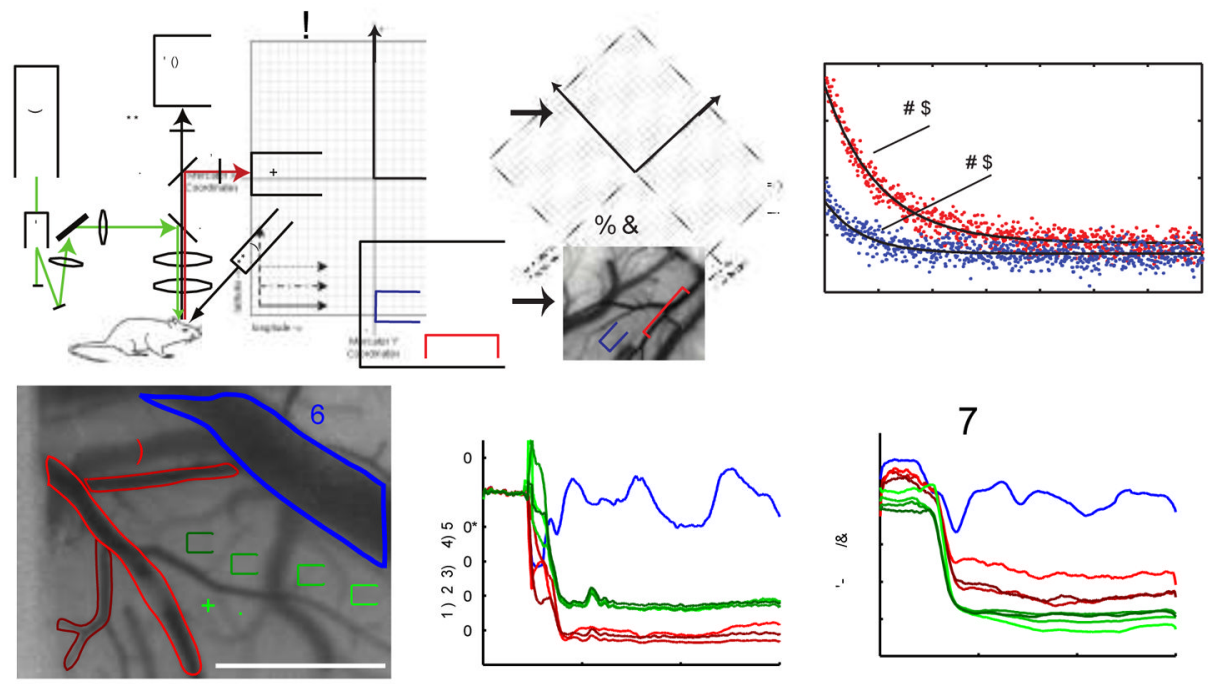

Figure 6.

(a)-(b) Combined instrument for simultaneous imaging of blood flow and spatial mapping of $\mathrm{pO}_{2}$. Spatially resolved $\mathrm{pO}_{2}$ is obtained by spatially restricting phosphorescence excitation light and utilizing a single element detector to record time-resolved phosphorescence decays (c). (d)-(f) Measured temporal dynamics of blood flow and $\mathrm{pO}_{2}$ in 8 different spatial regions following stroke induction at $t=100 \mathrm{~s}$. 